\title{
Des didactiques des disciplines scolaires à la didactique comme science anthropologique
}

Sur un obstacle épistémologique, psychologique et institutionnel

Yves Chevallard

\section{(2) OpenEdition}

\section{Journals}

Édition électronique

URL : http://journals.openedition.org/educationdidactique/1863

DOI : 10.4000/educationdidactique.1863

ISSN : $2111-4838$

\section{Éditeur}

Presses universitaires de Rennes

Édition imprimée

Date de publication : 30 juillet 2014

Pagination : $35-43$

ISSN : 1956-3485

\section{Référence électronique}

Yves Chevallard, «Des didactiques des disciplines scolaires à la didactique comme science anthropologique », Éducation et didactique [En ligne], 8-1 | 2014, mis en ligne le 15 septembre 2016, consulté le 20 avril 2019. URL : http://journals.openedition.org/educationdidactique/1863 ; DOI :

10.4000/educationdidactique.1863 


\section{DES DIDACTIQUES DES DISCIPLINES SCOLAIRES À LA DIDACTIQUE COMME SCIENCE ANTHROPOLOGIQUE SUR UN OBSTACLE ÉPISTÉMOLOGIQUE, PSYCHOLOGIQUE ET INSTITUTIONNEL}

Yves Chevallard

Université d'Aix-Marseille, EA 4671 ADEF, ENS de Lyon, IFE

La science, vous le savez, n'est pas une révélation, il lui manque longtemps après ses débuts ces caractères de certitude, d'immutabilité, d'infaillibilité auxquels la pensée humaine aspire si ardemment. Mais telle qu'elle est, elle est tout ce que nous pouvons avoir.

Sigmund Freud, La question de l'analyse profane 
Yves Chevallard

\section{INTRODUCTION}

Dans ce qui suit, je me réfère explicitement à la situation française, sans écarter pour autant la possibilité que mon propos vaille bien plus largement. Je le fais en prenant appui sur la théorie anthropologique du didactique, que je ne présenterai pas davantage $\mathrm{ici}^{1}$. Pour la précision de mon propos, j'ai utilisé sans façons les « petites lettres» dont parlait Lacan (2001). Je comprends le coût psychologique et culturel que cela peut faire peser sur le lecteur grammatophobe. Je demande cependant à ce dernier d'en méditer le bénéfice scientifique, auquel je ne saurais renoncer, au nom même de ce dont je parle ici.

\section{CONFUSIONS ET RUPTURES}

Ce que je nommerai désormais la didactique s'est constitué en partant des disciplines scolairement enseignées. Ainsi voit-on apparaître des didacticiens des mathématiques, des didacticiens $d u$ français, des didacticiens de l'EPS, etc. Dans ce qui suit, je noterai $\xi$ un chercheur en didactique, $\mathcal{D}$ une discipline scolaire (ou universitaire), $\xi_{\mathscr{D}}$ un didacticien « de $\mathscr{D} », y$ un professeur, $y_{\mathscr{D}}$ un professeur de $\mathscr{D}$ et $\partial_{\mathscr{D}}$ la didactique de $\mathscr{D}$, notant $\bar{\partial}$ ce que j'ai appelé la didactique, tout court (on a bien sûr $\partial_{\mathscr{D}} \subset \bar{\partial}$ ).

De façon plus ou moins subreptice, ce procédé de nomination de divers domaines de la science didactique, qui transmue un fait institutionnel en un fait épistémologique, cache - ou dévoile - l'assomption en général acritique, par nombre de didacticiens, de disciplines scolaires $\mathcal{D}_{1}, \mathscr{D}_{2}, \ldots, \mathscr{D}_{n}$ réputées « données », cette assomption allant souvent de pair avec une fidélité passionnée à la discipline $\mathcal{D}$ à laquelle $\xi_{\mathscr{D}}$ dit être attaché, ce qui le conduit fréquemment à assumer sans mot dire les contingences de la formation et des déformations historiques de disciplines dont les frontières elles-mêmes varient sous l'effet de faisceaux de contraintes qu'il échoit au didacticien, non de regarder comme allant de soi, mais d'étudier et d'expliquer ${ }^{2}$.

Ce choix « disciplinaire » bloqué tend à assigner au chercheur en didactique $\xi_{\Phi}$ le rôle ambigu de frère ou du moins de collègue du professeur $y_{\mathscr{D}}$, alter ego pour qui, comme il en va pour $y_{\mathscr{D}}$, la discipline $\mathcal{D}$ est un donné non problématique, qui ne serait problématique que pour les élèves, tandis que le reste du monde (soit... les professeurs et didacticiens des autres disciplines $\left.\mathscr{D}^{\prime} \neq \mathscr{D}\right)$ ne serait pas concerné. Il faut ajouter que, même quand le professeur ne le reconnaît pas pour tel, ce frère se conçoit comme un grand frère du professeur, son aîné, dans la mesure où il est supposé - d'abord par lui-même -, en savoir plus, par exemple déjà s'agissant de la discipline $\mathscr{D}$ (notamment quand le didacticien est un universitaire « spécialiste » de cette discipline). Cette position imaginaire de surplomb, utile pour en imposer et faire accepter par exemple les postures d'observateur et d'expérimentateur que le chercheur ne saurait manquer d'adopter régulièrement, a pourtant un coût scientifique fort élevé pour la didactique ${ }^{3}$.

La position de chercheur en didactique ne se constitue ni par contiguïté, ni par relèvement à partir de la position de professeur : elle suppose une rupture épistémologique avec cette position, afin que le chercheur puisse constituer l'univers du professeur (les connaissances enseignées, les élèves, etc.) en objet d'étude. Il va de soi que le chercheur $\xi$ n'est en rien « supérieur» au professeur $y$ ou en surplomb par rapport à lui. Leurs positions respectives sont en quelque sorte incommensurables: le topos du chercheur $\xi$ n'est pas celui du professeur $y$, et réciproquement. Il résulte de là en particulier (a) que le chercheur en didactique $\xi$ n'a nullement à reprendre à son compte les contraintes qui pèsent ordinairement sur le professeur (mais il a, en revanche, à les étudier et à les expliquer), et (b) que le chercheur $\xi$ doit assumer des contraintes propres à la position qu'il prétend occuper, contraintes qu'il doit pour cela identifier et analyser.

\section{INCLUSIONS ET EXCLUSIONS}

Formellement, le chercheur $\xi$ étudie une question $Q$ à laquelle il s'efforce (en général en équipe) d'apporter une réponse $\mathbb{R}$. Parmi les contraintes que $\xi$ doit assumer, il y a évidemment celles portées par les objets que mentionne la question $Q$. Parmi ces objets, il y a ce qui, dans l'institution « didactique » $I$ considérée dans la question $Q$, est regardé comme enjeu didactique. Cet enjeu, qui est une ouvre (soit une production humaine réputée délibérée), est dénoté par le symbole (« cœur »). Les enjeux didactiques sont généralement regardés comme relevant d'une discipline $\mathcal{D}$, ou d'un certain domaine $\Delta$ à l'intérieur de celle-ci, ou d'un secteur $\sum$ de ce domaine, etc. Tout cela impose à l'œuvre $\boldsymbol{\vee}$, dans $I$, des contraintes qui 
sont aussi des contraintes pesant sur l'étude et l'apprentissage de $\boldsymbol{\varphi}$. S'agissant d'une institution $I$ participant du système scolaire courant dans la société française actuelle (écoles, collèges, lycées, universités), on doit mettre en avant les deux contraintes suivantes, généralement laissées inanalysées, ou du moins passées sous silence, mais qui se révèlent décisives dans l'abord du problème examiné ici.

a) Si l'enjeu didactique $\boldsymbol{\bullet}$ est reconnu, dans la culture de $I$, comme "appartenant " à la discipline $\mathscr{D}$, alors les professeurs $y_{\mathscr{D}}$ sont légitimes pour « enseigner " l'œuvre $\boldsymbol{\bullet}$, et cela quelle que soit leur « compétence » réelle pour ce faire. Ils conservent cette légitimité même lorsque, l'œuvre étant par exemple nouvellement introduite dans le corpus à enseigner, le didacticien $\xi$ ou d'autres observateurs savent que, dans un premier temps, cette compétence fera défaut aux professeurs $y_{\mathscr{D}}$.

b) Les professeurs $y_{\mathscr{D}}$ sont seuls légitimes pour « enseigner » l'œuvre $\boldsymbol{\vee}$. Lorsqu'un professeur $y_{\mathcal{D}^{\prime}}$ (où $\mathscr{D}^{\prime} \neq \mathscr{D}$ ) recourt avec sa classe à l'œuvre $\boldsymbol{\vee}$, si du moins ce recours n'est pas tout simplement nié, cela conduit à regarder comme un adjuvant, voire comme un simple excipient de la discipline $\mathcal{D}^{\prime}$. La spécificité supposée de l'usage de $\boldsymbol{}$ 《dans » $\mathscr{D}^{\prime}$ tend ainsi à gommer l'appartenance de $\boldsymbol{V}$ à $\mathscr{D}$ parce que

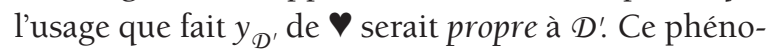
mène va de pair avec le déni de didacticité, relativement à $\boldsymbol{\vee}$, des situations de classe traversées : l'œuvre $\checkmark$ ne pouvant être assumée dans $\mathscr{D}^{\prime}$ comme enjeu didactique, elle cesse du même coup d'y être réputée problématique et, au contraire, y est bientôt tenue comme « allant de soi ».

Lorsqu'il ne conduit pas tout simplement à leur éviction pure et simple (ce qui est fréquemment le cas s'agissant des outils mathématiques par exemple), le phénomène de la dénégation de l'appel par $D^{\prime}$ à des outils extra-disciplinaires réduit la qualité du rapport à ces outils et aboutit souvent à engendrer un rapport indiscipliné ou, du moins, mal discipliné, et pour cela à la fois insuffisamment intelligible et créant peu d'intelligibilité dans $\mathcal{D}^{\prime}$. Pour ne donner ici que l'exemple d'une notion aujourd'hui encore largement « profane » (c'est-à-dire non intégralement revendiquée par une discipline scolaire qui prétendrait en avoir l'exclusivité), la notion d'effet de serre, souvent présentée dans une contiguité commode avec le fonctionnement d'une serre de jardin ${ }^{4}$, ignore en conséquence la question de la physique de l'effet de serre, ce qui, entre autres choses, conduit à ignorer l'un des points d'attaque de certains climato-sceptiques contre le fait du réchauffement climatique ${ }^{5}$.

L'absence ou l'insuffisance de la rupture épistémologique entre $\xi$ et $y$ et la contiguité maintenue de leurs positions respectives font alors que les contraintes précédemment énoncées sont « transférées » des professeurs aux didacticiens : le didacticien de la discipline $\mathscr{D}$ (a) est censé légitime a priori pour étudier toute question $Q$ relative à l'enseignement et à l'apprentissage de $\mathscr{D}$, c'est-à-dire toute question relevant de $\partial_{\mathscr{D}}$; et (b) est regardé comme illégitime pour étudier toute question $Q^{\prime}$ relative à $\partial_{\mathscr{D}^{\prime}}-$ à moins que $\xi_{\mathscr{D}}$ ne soit en même temps regardé comme « didacticien de $\mathscr{D}^{\prime}$ », ce qui est, aujourd'hui encore, rare. Cela soulève à l'évidence la question de ce qui qualifie un chercheur en didactique $\xi$ comme didacticien d'une discipline $\mathcal{D}$.

\section{LA FABRIQUE DES DIDACTICIENS}

À partir de la fin des années 1960, un didacticien de $\mathscr{D}$, où $\mathscr{D}$ est une discipline scolaire, est généralement une personne ayant reçu une formation supérieure dans la discipline $\mathcal{D}$ et, préférentiellement, une personne qui enseigne ou a enseigné $\mathcal{D}$ soit au secondaire (au sens large, y compris en école normale), soit à l'université, et est généralement lauréate du CAPES ou de l'agrégation de $\mathscr{D}$ (pour autant que de tels concours de recrutement existent; mais la description proposée ici vaut, mutatis mutandis, pour toutes les disciplines). Cette genèse des communautés de didacticiens explique sans doute que les valeurs propres aux corporations de professeurs aient migré souvent ne varietur vers les didacticiens. Ainsi en va-t-il notamment de l'attachement passionné à la discipline $\mathscr{D}$, qui tend à faire du professeur $y_{\mathcal{D}}$ un gardien ombrageux de «sa » discipline, capable de surgir à tout moment pour la défendre contre les étrangers à la tribu qui voudraient ne serait-ce qu'exprimer un avis sur $\mathcal{D}$ et son enseignement. On retrouve beaucoup de ce sentiment de propriété chez les didacticiens $\xi_{\mathscr{D}}$, ce qui institue un interdit efficace pour scinder la science didactique $\bar{\partial}$ en domaines « disciplinaires $» \partial_{\mathscr{D}}$ aux frontières quasi imperméables séparant l'autochtone de l'allochtone, le citoyen de plein exercice du forain équivoque.

La scission historique du champ de la didactique selon le patron du système des disciplines scolaires doit être interrogée au plan scientifique. On notera 
d'abord ceci : puisque l'intervention d'un didacticien $\xi$ tenu pour un $\xi_{\mathscr{D}}$ dans la didactique de $\mathscr{D}^{\prime}\left(\right.$ avec $\mathscr{D}^{\prime} \neq$ $D$ ) est regardée comme illégitime et se trouve, peu ou prou, « interdite », ou du moins non prise en compte, le « débat scientifique » dans $\partial_{D^{\prime}}$ n'est ouvert qu'aux « indigènes » de la discipline $\mathcal{D}^{\prime}$, qui, de par leur origine commune, partagent largement les mêmes valeurs, les mêmes présupposés, les mêmes orthodoxies, ce qui ne manque pas de réduire et l'ampleur et la profondeur de ce débat pourtant crucial en une communauté scientifique. Bénéfice secondaire de l'opération : en excluant ceux qu'ils considèrent comme allochtones, un moindre nombre de gens - qui se regardent, quant à eux, comme autochtones - ont à se répartir l'autorité au sein de $\partial_{\mathbb{D}^{\prime}}$, ce qui engendre de tranquilles sinécures et, parfois, quelques caciques furieux. Au reste, ce stratagème est employé au sein même de $\partial_{\mathscr{D}^{\prime}}$, quand d'aucuns y délimitent la didactique $\partial \Delta$ d'un certain domaine $\Delta$ de $\mathscr{D}$, voire la didactique $\partial_{\Sigma}$ d'un certain secteur $\sum$ de $\Delta$, etc., en tentant de reproduire alors, autour de $\partial_{\Delta}$ ou de $\partial_{\Sigma}$, les barrières d'octroi déjà érigées autour de $\partial_{\mathscr{D}^{\prime}}$. Il faut ajouter à cette fermeture programmée du débat scientifique un autre travers fâcheux : ce qu'un didacticien $\xi$ peut faire alors, dans le champ $\bar{\partial}$ de la didactique, dépend de son passé de façon à peu près irréversible si l'on a fait des études initiales poussées de ceci, il est rare qu'on ait fait aussi des études poussées de cela ${ }^{6}$. Il y a là, sous couleur de contrôle épistémologique, un contrôle au faciès et à d'autres signes extérieurs d'extranéité (telles certaines manières de dire, par exemple). «Didacticien, vos papiers! » semble être ici l'interpellation dirimante. Le débat laisse place au non-dit, au supposé indicible, qui s'entend entre gens de la tribu mais resterait impénétrable à l'étranger.

\section{LE TERRITOIRE DU DIDACTICIEN}

Pour suggérer la déperdition qu'engendre le blocage actuel sur des didactiques qui se contentent de reproduire le système des disciplines scolaires et imposent fermement, voire furieusement, de détestables frontières, il convient d'en dire un peu plus sur la didactique. La didactique est la science dont l'objet premier est le didactique, c'est-à-dire l'ensemble des faits didactiques. Il y a fait didactique (ou " geste» didactique) dès lors qu'une instance $U$ (et par instance on entend ici une personne ou une institution) fait quelque chose ou même envisage de faire quelque chose pour aider à faire qu'une instance $V$ intègre à son équipement de savoir et de savoir-faire - à son équipement praxéologique - certaines connaissances relatives à l'enjeu $\boldsymbol{\nabla}$ du geste didactique.

L'étude du didactique et de ses effets d'apprentissage conduit alors à élargir le champ de l'étude à des ensembles de conditions étagées selon les niveaux de l'échelle de codétermination didactique reproduite ci-après - en ne retenant ici que le cas où les systèmes didactiques $S(X ; y ; \boldsymbol{V})$, où $X$ désigne les élèves et $y$ le professeur, sont "disciplinaires », c'est-à-dire où l'œuvre est regardée comme « appartenant » à une discipline $\mathscr{D}$ :

Humanité
$\uparrow \downarrow$
Civilisations
$\uparrow \downarrow$
Sociétés
$\uparrow \downarrow$
Écoles
$\uparrow \downarrow$
Pédagogies
$\uparrow \downarrow$
Disciplines
$\uparrow \downarrow$
Systèmes didactiques

L'idée cardinale est que chacun des échelons - des niveaux - est le siège de conditions et de contraintes qui sont susceptibles d'influer sur l'écologie et (donc) sur l'économie du didactique, lequel est présent dans des formes variées du haut en bas de l'échelle. Ce sont ces conditions et contraintes que l'on qualifie de didactiques, la spécificité des conditions didactiques croissant lorsqu'on descend l'échelle, leur généricité croissant lorsqu'on la monte.

Pour chaque enjeu didactique $\boldsymbol{\vee}$, le didacticien $\xi$ qui étudie une question $Q$ relative à l'enseignement et à l'apprentissage de $\boldsymbol{}$ devra reprendre l'analyse des conditions et contraintes jouant un rôle dans le phénomène sur lequel porte la question $Q$. Le fait pour un didacticien $\xi$ d'étudier une question $Q$ à propos d'un enjeu didactique suppose ainsi qu'il prenne en compte, pour y analyser les conditions pertinentes, l'ensemble des niveaux de codétermination didactique. Le didacticien $\xi$ devra donc se risquer 
à conduire des analyses didactiques à des niveaux « élevés » de l'échelle, sans être pour cela affilié à une quelconque tribu qui vivrait spécifiquement en tel ou tel niveau : $\xi$ devra faire des analyses pédagogiques, des analyses scolaires, des analyses sociales, etc., sans être pour autant "pédagogue », "spécialiste » de l'institution scolaire, sociologue, etc. Ses analyses devront - c'est sa spécificité et sa singularité parmi les éventuels «spécialistes » du niveau considéré porter sur les conditions qui affectent le didactique relatif à l'œuvre (et qui, en retour, sont affectées par lui). Au niveau des systèmes didactiques $S(X$; $y ; \boldsymbol{\vee}), \xi$ devra ainsi procéder à des analyses portant sur $X$ et ses membres $x$, sur $y$ aussi et, bien entendu, sur l'œuvre $\boldsymbol{\varphi}$. Comment cela est-il censé être (ou devenir) possible?

\section{LE CHAMP DE LA DIDACTIQUE ET LE DIDACTICIEN GYROVAGUE}

D’une manière générale, la réponse à la question précédente tient en peu de mots : c'est à la didactique $\bar{\partial}$ en tant que science en construction d'apporter la réponse attendue. Les analyses portant sur le niveau de la société, par exemple, sont des analyses « didacticiennes », qui peuvent bien recourir à des outils de natures diverses - empruntés à la sociologie, à l'histoire, à l'anthropologie, à la science politique, à la statistique, par exemple - mais qu'il convient d'élaborer de façon spécifique et qu'on ne saurait espérer, sinon par miracle, trouver ready-made en d'autres champs scientifiques dès lors qu'il s'agit d'analyses didactiques de certaines conditions et contraintes spécifiques de la question Qétudiée. Que doit donc savoir $\xi$ pour être à la hauteur de sa tâche ? Là encore, c'est à la didactique de répondre, non à quelque puissance tutélaire qui la surplomberait.

Considérons le cas décisif de l'analyse des conditions et contraintes portées, au sein du système didactique $S(X ; y ; \boldsymbol{\vee})$, par l'enjeu didactique $\boldsymbol{\vee}$. Ce que $\xi$ doit savoir - ou plutôt : doit se donner la liberté d'apprendre - à propos de l'œuvre ne saurait a priori être identifié à ce que sait, en moyenne, le professeur $y$ de la discipline $\mathscr{D}$. En revanche $\xi$ devra souvent se mettre en peine d'identifier ce que sait $y$ à propos de $\boldsymbol{\vee}$, ce qu'un élève $x \in X$ sait et ce qu'il est censé apprendre, quelles relations l'œuvre $\boldsymbol{}$ entretient avec d'autres enjeux didactiques $\boldsymbol{}$ * affirmés ou, au contraire, plus ou moins niés dans la classe
$C(X ; y ; \mathcal{D})$ où se forme le système didactique $S(X ;$ $y ; \boldsymbol{V})$, etc. C'est à $\xi$ d'abord, à l'équipe $\Xi$ à laquelle il appartient ensuite, de répondre. Pour ce faire, $\xi$ ou plutôt $\Xi$ devra élaborer un modèle praxéologique $\operatorname{MP}(\Xi, \boldsymbol{\vee})$ pour $\Xi$ de l'œuvre $\boldsymbol{\vartheta}$. Une telle élaboration suppose que cette œuvre soit située par ces chercheurs à l'intérieur de ce que je nommerai ici un système de connaissances $S$, le didacticien $\xi$ et l'équipe de recherche $\Xi$ travaillant alors à construire, de facto, avec d'autres didacticiens, une didactique de $S$, soit $\partial_{S}$, à l'intérieur de laquelle sera produite la réponse $\mathbb{R}$ à donner à la question Qétudiée ${ }^{7}$.

On prendra garde, à cet égard, aux pièges de l'homonymie : si, par exemple, $\boldsymbol{\nabla}$ appartient au domaine $\mathcal{A}$ de l'algèbre élémentaire au sens du collège, la didactique « de l'algèbre » (ou de tel secteur de « l'algèbre $»$ ), soit $\partial_{S}$, où $S$ est un « système de connaissances » au sens évoqué plus haut, ne se superposera exactement ni avec ce que sait $y$ ni avec ce que $x \in X$ devra avoir appris en fin d'étude en matière d'« algèbre » $\mathcal{A}$. En outre, deux équipes de didacticiens, travaillant indépendamment sur la même question $Q$ relative à $\boldsymbol{\nabla}$, pourront identifier deux systèmes de connaissances $S$ et $S$ 'quelque peu différents et travailler ipso facto à construire deux didactiques $\partial_{S}$ et $\partial_{S^{\prime}}$ qui ne soient pas en tout point consonantes - à charge pour le débat scientifique au sein de $\bar{\partial}$, nourri par l'ensemble des travaux pertinents réalisés dans $\bar{\partial}$, de tracer un chemin non déterminé d'avance conduisant à la réduction des dissonances éventuelles entre $\partial_{S}$ et $\partial_{S^{\prime}}$.

Le travail des didacticiens à propos d'un enjeu didactique les conduit ainsi à construire des entités qui ne sont pas les simples copies du « donné » institutionnel étudié, mais qui permettent de modéliser et d'interroger ce « donné » : pourquoi apparaît-il en telle société, en tel type d'écoles, en tel type de systèmes didactiques? Comment telle pédagogie estelle censée conduire les élèves jusqu'à l'œuvre $\boldsymbol{V}$ ? Que peut faire $x \in X$ pour étudier (et donc « apprendre ») $\checkmark$ et comment $y$ peut-il l'y aider ? Quelles conditions et contraintes liées à l'environnement « disciplinaire » de apparaissent-elles susceptibles d'influencer l'écologie et l'économie didactiques de ? À toutes ces questions, et à beaucoup d'autres encore, les connaissances d'un $y_{\mathcal{D}}$ relatives à l'œuvre ne peuvent à coup sûr suffire à apporter réponse, en même temps qu'elles ne sont pas toutes nécessaires pour cela. Par principe de méthode, le didacticien doit alors reprendre l'étude $a b$ ovo, afin de 
Yves Chevallard

construire et exploiter le milieu M des outils d'étude et de recherche indispensables pour construire et valider la réponse $R$ qu'il s'efforce de produire.

Pour préciser encore les choses, j'ai introduit récemment ce qui est d'abord un personnage, qui peut s'actualiser en diverses personnes, certes, mais qui reste en premier lieu une figure emblématique : celle du didacticien gyrovague, noté ici $\xi^{\gamma}$ parce que la lettre $\gamma$ est l'initiale du grec yôpoৎ « cercle», d'où vient le latin ancien gyrus « cercle», le mot gyrovague (composé de gyrus et de vagus « vagabond ») désignant à l'origine un moine itinérant, n'ayant pas de monastère propre mais allant de monastère en monastère (genre de vie monastique qu'interdit en 451 le concile de Chalcédoine). Contrairement aux didacticiens dont l'affiliation disciplinaire semble être l'alpha et l'oméga, le didacticien gyrovague n'a pas d'affiliation disciplinaire connue et se garde de rien faire connaître sur ce chapitre. Au fil de ses cheminements de recherche, il étudie avec maestria - le personnage est de fiction, rêvons donc un peu ! - des questions de didactique à propos d'enjeux didactiques relevant éventuellement de disciplines diverses, en sorte que ses interventions pourraient le faire passer, tour à tour, pour affilié à une pluralité de disciplines $\mathscr{D}, \mathscr{D}^{\prime}, \mathscr{D}^{\prime \prime}$, etc. Au cours de ses pérégrinations de recherche, le didacticien gyrovague $\xi^{\gamma}$ ne se laisse pas aller à des connivences « disciplinaires » avec les didacticiens avec qui il travaille, qui sont souvent des didacticiens $\xi, \xi_{\mathbb{D}^{\prime}}$, $\xi_{\mathbb{D}^{\prime \prime}}$ etc., disciplinairement affiliés et, quelquefois, confinés. Il ne permet pas qu'on l'interroge là-dessus, n'offrant au débat scientifique que les réponses $R$ qu'il bâtit et les arguments avec lesquels il les défend.

\section{LE MÉTIER DE DIDACTICIEN}

Semblable didacticien $\xi^{\gamma}$ peut-il exister ? Avant de répondre, il convient de s'arrêter sur un argument qu'on peut vouloir élever contre l'abaissement des frontières au sein du champ $\bar{\partial}$ de la didactique. Un tel affaissement, penseront certains, pourrait conduire, sinon jusqu'au type pur du didacticien gyrovague, du moins à l'apparition de didacticiens " multispécialisés », jugés pour cela inemployables par des institutions résolument monodisciplinées. Il y a là sans doute un argument sincère, qui s'inspire d'une réalité où, volens nolens, plus d'un didacticien se trouve aujourd'hui enfermé. Il s'agit pourtant, me semble-t-il, d'un paralogisme périlleux pour le métier de didacticien. Voyons cela.

Imaginons pour l'occasion la situation suivante (qui, aujourd'hui, n'existe encore que très peu) : le métier de didacticien s'exerce dans des instituts de didactique, dans des laboratoires de didactique, dont chacun peut avoir ses domaines de recherche de prédilection, d'où le didacticien s'extrait pour « faire du terrain » en différents lieux institutionnels et, bien sûr, pour participer à la vie collective de sa communauté scientifique. Ce schéma, qui rapproche la figure du didacticien de celle du sociologue, du psychologue, de l'ethnologue, de l'écologue, etc., comporte évidemment des dangers, notamment celui de voir se développer une didactique " libérale ", innocente de tout engagement institutionnel, social et politique, prise aux rets de ses seules logiques internes. Mais la situation actuelle - telle que je la perçois - a des effets peut-être plus dommageables encore.

Nombre de didacticiens, fera-t-on donc observer, ne trouvent actuellement d'emploi que dans des institutions de formation de professeurs. Pour la simplicité de l'argument, tenons-nous-en ici à la formation de professeurs de l'enseignement secondaire, dans la mesure où ceux-ci sont regardés comme des spécialistes de l'enseignement d'une discipline $\mathscr{D}$ donnée. L'institution de formation exige alors, en tant qu'employeur, que le didacticien $\xi$ soit certifié « didacticien $\xi_{\mathscr{D}}$ ", ce qui, dans le système disciplinaire étanche aujourd'hui régnant, requiert qu'il ait, dans la discipline $\mathcal{D}$, une formation au moins équivalente, en extension comme en profondeur, à celle d'un professeur $y_{\mathscr{D}}$, par exemple parce qu'il serait à l'origine un tel professeur « certifié ». Ce jeu de rôles constitue, pour les deux parties contractantes, un marché de dupes. Celui que l'institution de formation recrute n'est trop souvent qu'un formateur de professeurs qui a un diplôme de didacticien (un master ou une thèse par exemple). Mais elle ne recrute pas un didacticien (de $\mathscr{D}$ ou d'autre chose) $)^{8}$. Le recruté ne l'est pas en tant que didacticien mais en tant qu'ayant reçu aussi une formation en didactique (de $\mathscr{D}$ ), ce qui, aux yeux de quelques-uns, ennoblit la fonction de base pour laquelle on le recrute et lui permet en principe de donner un enseignement " de didactique » trop souvent réduit à la visite de quelques " œuvres » didactiques connues.

Peut-on rompre avec ce marché de dupes ? Assurément. Mais soulignons d'abord que c'est là un problème posé au métier de didacticien, à travers 
des individus qui tentent de l'exercer. Si l'on écarte la figure du didacticien qui répond à la demande de l'institution tout en conservant comme il le peut des thèmes de recherche sans lien avec elle et qui n'est donc pas un didacticien de l'institution qui l'emploie, lorsqu'un didacticien $\xi$ est recruté par une institution de formation professionnelle, sa mission est de contribuer, par la recherche et par l'enseignement, à résoudre les problèmes de la profession à laquelle prépare cette institution - ce qui devrait être, au demeurant, un critère essentiel de son recrutement ${ }^{9}$. En d'autres termes, $\xi$ doit se donner pour terrain, de façon non nécessairement exclusive, certes, les problèmes de didactique soulevés par la formation professionnelle située au cœur de l'institution qui le recrute. Par contraste, le recrutement d'un didacticien qui ne peut assumer pleinement son métier de didacticien fait trois sortes de victimes : le métier de didacticien, l'institution de formation (et le métier de formateur correspondant), enfin la profession même autour de laquelle l'institution de formation s'est constituée. Il appartient donc à la profession de didacticien de travailler à faire reconnaître par les institutions de formation de professeurs d'autres critères de recrutement de didacticiens que les critères qui président aujourd'hui au recrutement de professeurs - ce qui ne saurait manquer de faire évoluer le recrutement des professeurs lui-même. En particulier, un didacticien $\xi$ « de la discipline $\mathcal{D} »$, ne devrait pas se voir prioritairement tenu d'enseigner aux personnes en formation la discipline $\mathcal{D}$, tout court. En revanche, l'une de ses missions en tant qu'enseignant-chercheur didacticien dans une institution de formation de professeurs devrait avoir pour objet la déconstruction et la reconstruction - en synchronie et en diachronie - des modèles praxéologiques du type $\operatorname{MP}\left(\boldsymbol{\vee}, y_{\mathscr{D}}\right)$, où $y_{\mathcal{D}}$ est un professeur de la discipline $\mathcal{D}$, ce qui revient grosso modo à réaliser ces opérations sur les modèles praxéologiques $\operatorname{MP}(\boldsymbol{\nabla}$, $\left.\hat{Y}_{\mathscr{D}}\right)$, où $\hat{Y}_{\mathscr{D}}$ est le métier de professeur de $\mathscr{D}$. Une telle disjonction devrait permettre de distinguer, en droit comme en fait, la spécificité de l'équipement praxéologique touchant la discipline $\mathscr{D}$ qui doit être celui d'un didacticien $\xi$ agissant comme « didacticien de $\mathcal{D} »$, et cela tant en matière de recherche qu'en matière d'enseignement et de formation ${ }^{10}$.

Un tel didacticien $\xi^{\gamma}$ peut-il donc exister ? Pour qu'une réponse positive puisse être donnée à cette question cruciale pour le devenir du métier de didacticien, plusieurs conditions cardinales doivent être réalisées par la communauté des didacticiens qui refusent de s'enfermer dans leur castrum disciplinaire en cédant à une tentation obsidionale prégnante encore aujourd'hui. Une condition clé, fort prosaïque sans doute, est que cette communauté œuvre à créer des infrastructures appropriées, en offrant par exemple à ses membres (a) en fonction de leurs projets de recherche, des formations en mathématiques, en génétique, en physique, en linguistique, en arts plastiques, etc., pour didacticiens, « tout court », et (b) en fonction de leurs projets professionnels, des formations disciplinaires ou codisciplinaires pour les didacticiens visant à occuper tel ou tel type de positions institutionnelles.

Une autre condition nécessaire que nous devons viser consiste en l'émergence d'une conscience didacticienne commune et assumée, qui nous rassemble, quand l'entre-soi des consciences disciplinaires divise. La didactique a beaucoup pâti de ce qu'on s'interdise et qu'on interdise de parler d'elle à la façon dont on parle sans ambages de la physique, de la sociologie, de la biologie, de la psychologie, etc. Sous l'empire d'un dogmatisme disciplinaire épistémologiquement « ethniciste », on a jusqu'ici fragmenté $\bar{\partial}$ en morceaux prétendument incommensurables, monades qui ne dialoguent qu'avec elles-mêmes et ne forment pas ensemble un univers susceptible de participer à la vie de la cité scientifique à l'instar des autres sciences, si débutantes soient-elles. Désigner $\bar{\partial}-l a$ didactique - comme ayant une unité - qu'elle tend depuis l'origine à réaliser spontanément, contre les résistances « disciplinaristes »- est nécessaire pour lui donner, dans la vie académique comme dans la vie culturelle et institutionnelle, une présence visible, remarquée, le cas échéant discutée, mais affirmée.

\section{CONCLUSION}

La définition de la didactique des mathématiques avancée jadis par Guy Brousseau, selon laquelle celle-ci « est la science des conditions spécifiques de la diffusion des connaissances mathématiques » (Brousseau, 2010, p. 1 : c'est moi qui souligne), a joué un rôle vital pour ouvrir une brèche dans l'indifférence aux contenus d'une certaine " pédagogie » traditionnelle; mais elle a du même coup joué un rôle non négligeable dans le repli identitaire que nous devons aujourd'hui combattre. Cette définition doit être renouvelée : la didactique est la science des 
conditions de la diffusion des connaissances dans les institutions d'une société. Et ce que cette science dit au monde qui nous entoure, c'est que les conditions explicatives de leur diffusion (ou de leur nondiffusion) comportent toujours et des conditions très spécifiques des connaissances considérées, et des conditions plus génériques, qui s'étagent en une succession de niveaux, obligeant ainsi le didacticien à se faire anthropologue, sociologue, pédagogue, psychologue, etc., de la diffusion des connaissances s'il veut être vraiment didacticien.

\section{NOTES}

1. Pour quelques indications, voir Chevallard, 2010.

2. Une contrainte relativement à une position institutionnelle $p$ est une condition qu'on ne saurait modifier, du moins à court terme, en tant qu'on occupe la position $p$.

3. L'assujettissement à la discipline $\mathscr{D}$, quand il n'est pas compensé par des contre-assujettissements appropriés, ne permet guère de se former une vision de $\mathscr{D}$ comme objet historico-social par exemple. Ainsi cela ne permet-il guère de voir - pour prendre ici un fait au centre de travaux actuels de l'auteur - que les mathématiques enseignées sont aujourd'hui encore le vestige d'une construction curriculaire ancienne destinée à la formation des futures élites scientifiques, projet sociopolitique légitime en lui-même mais qui a depuis longtemps aboli la tradition mathématique «populaire » propre à l'ancien ordre primaire. L'élaboration de contre-assujettissements permettant de réduire l'angle mort dû aux assujettissements professoraux et autres constitue une composante décisive du travail « didacticien ».

4. Voir ainsi Gouvernement du Canada, 2012. L'expression d'effet de serre aurait été introduite par Alexander Graham Bell (1847-1922), l'inventeur du téléphone, dans un article de 1917, où l'auteur affirme que l'usage incontrôlé des carburants fossiles pourrait engendrer « a sort of greenhouse effect » (voir Alexander Graham Bell, s. d.).

5. Voir par exemple, à ce propos, Connolley, 2011.

6. On pourrait montrer, au demeurant, que prétendre le faire serait, dans une majorité de cas, une ambition stérile. 7. L'expression «système de connaissances » désigne en ce cas, et pour faire court, un ensemble de connaissances $S$ formant un système relativement autonome à l'intérieur d'un sur-ensemble de connaissances $\check{S}$ suffisamment vaste, en cela que 1) $S$ contient les connaissances de $\breve{S}$ nécessaires pour fonder les connaissances présentes dans $S$, et 2) $S$ contient les connaissances qu'il permet d'engendrer dans $\check{S}$. En d'autres termes, $S$ est fermé dans $\check{S}$ pour les chaînes trophiques de connaissances. À titre d'illustration, on peut songer à l'exemple, évoqué plus haut, de la notion d'effet de serre : en ce cas, $S$ pourrait avoir à contenir entre autres des connaissances relatives aux apports non seulement de Bell (1917), mais aussi bien de Joseph Fourier (1824), de John Tyndall (1859), de Svante Arrhenius (1896), etc. La délimitation - toujours quelque peu floue - de Š est bien entendu une opération cardinale, vouée pour cela à être indéfiniment reprise, dans la définition d'un système de connaissances $S$.

8. La confusion des rôles qui, à cet égard, était observable dans les IUFM semble promise à un avenir durable : on 
peut douter qu'elle diminue avec la substitution des ESPE (Écoles supérieures du professorat et de l'éducation) aux IUFM.

9. Voir Chevallard et Cirade, 2009 ainsi que Chevallard et Cirade, 2011.

10. Le problème de la faiblesse actuelle et de la didactique $\bar{\partial}$, et de l'existence assumée de toute gyrovaguie didacticienne resurgit au sein même des institutions de formation de professeurs. Supposons en effet qu'on y enseigne un tant soit peu la didactique de $\mathcal{D}$, soit donc la discipline $\partial_{\mathscr{D}}$. L'institution devra, par suite, affronter des questions d'enseignement et d'apprentissage de la discipline $\partial_{\mathscr{D}}$. Qui étudiera alors ces problèmes de didactique de la didactique de $\mathcal{D}$ ? Selon les notations introduites plus haut, et par définition, des didacticiens $\xi_{\partial \mathcal{D}}$. Seront-ils les mêmes que les didacticiens $\xi_{\mathscr{D}}$ que l'institution aura recrutés ? Ou bien faudra-t-il recruter de « vrais » didacticiens $\xi_{\partial \mathcal{D}}$ ? Ou encore, faute de tels « spécialistes », niera-t-on ces problèmes didactiques-là, comme si la didactique de $\mathcal{D}$ suffisait à résoudre les problèmes de didactique de la didactique de $\mathcal{D}$, ainsi que d'aucuns l'ont si longtemps maintenu - implicitement en général, mais quelquefois explicitement - s'agissant de la discipline $\mathcal{D}$ elle-même, regardée par eux comme recelant tous les moyens souhaitables pour élucider les difficultés rencontrées par sa propre diffusion ?...

\section{RÉFÉRENCES}

Alexander Graham Bell. (s. d.). Dans Wikipedia. Récupéré le 10 décembre 2013, de

http://fr.wikipedia.org/wiki/Alexander_Graham_Bell

Brousseau, G. (2010). Glossaire de quelques de quelques concepts de la théorie des situations didactiques en mathématiques (1998). Récupéré le 10 décembre 2013, de

http://guy-brousseau.com/wp-content/uploads/2010/09/ Glossaire_V5.pdf

Chevallard, Y. (2010). La didactique, dites-vous ? Éducation \& didactique, 4(1), 139-146.

Chevallard, Y. \& Cirade, G. (2009). Pour une formation professionnelle d'université. Éléments d'une problématique de rupture. Recherche et formation pour les professions de l'éducation, 60, 51-62.

Chevallard, Y. \& Cirade, G. (2010). Les ressources manquantes comme problème professionnel. Dans G. Gueudet \& L. Trouche (Éds), Ressources vives. Le travail documentaire des professeurs en mathématiques (p. 41-55). Rennes: PUR et Paris : INRP.

Connolley, W. M. (2011). The greenhouse effect is not the effect that warms greenhouses. ScienceBlogs. Récupéré le 10 décembre 2013, de

http://scienceblogs.com/stoat/2011/07/19/the-greenhouseeffect-is-not-t/

Gouvernement du Canada. (2012). Effet de serre. Laction du Canada sur les changements climatiques. Récupéré le 10 décembre 2013, de

http://climatechange.gc.ca/default.asp?lang=Fr\&n= 1A0305D5-1

Lacan, J. (2001). Létourdit. Autres écrits (p. 449-495). Paris : Le Seuil. 
